\title{
SERVICE DISCOVERY PROTOCOL IN PROACTIVE MOBILE AD HOC NETWORKS
}

\author{
$\mathrm{M}^{\mathrm{a}}$ Isabel Vara ${ }^{1}$, Jose $\mathrm{M}^{\mathrm{a}}$ Cabero ${ }^{1}$, Jose Luis Jodrá ${ }^{2}$ and Jose Oscar Fajardo ${ }^{2}$ \\ ${ }^{I}$ Fundación Robotiker, Parque Tecnológico de Zamudio, edificio 202, 48170 Zamudio; ${ }^{2}$ UPV- \\ EHU University, Alameda Urquijo s/n, 48013 Bilbao
}

\begin{abstract}
This paper proposes a service discovery protocol for discovering and advertising services in a proactive ad hoc network. The protocol we have defined is piggybacked into the OLSR protocol. We define a new message type into OLSR called Service Discovery Message (SDM) for both advertisement and discovery of services. The advertisement frequency and advertisement lifetime are user-controlled parameters, so that they can be modified depending on the user requirements. Each node maintains a service cache to store information about its own services, and the services each device discovers in the network. We also present simulation results of our protocol and show that the service discovery protocol defined here achieves much efficiency in discovering services, while it introduces practically no packet overhead compared to the basic OLSR protocol.
\end{abstract}

Key words: Ad hoc networks; OLSR protocol; Service Discovery.

\section{INTRODUCTION}

Service discovery enables users, network devices and applications to seek out and find other users, networks devices and applications, in a friendly and easy way. There are some implementations that take this into account: Service Location Protocol (SLP) ${ }^{1}$ of IETF, Sun's Jini', Microsoft's Universal Plug and Play $(U p N P)^{3}$ and Bluetooth's Service Discovery Protocol $(S D P)^{4}$. However, most of them are only suitable for traditional fixed IP based networks. 
The main focus of this paper is to present a service discovery protocol integrated into $\mathrm{OLSR}^{5}$ protocol, to support service discovery and advertisement in ad hoc networks.

OLSR is a proactive ${ }^{6}$ protocol. Proactive protocols attempt to periodically evaluate all available routes within a network, so that when a packet needs to be forwarded, the route is already known and can be used immediately. The reason for integrating service discovery with a proactive routing protocol is that, this way the network efficiency increases because, the same routes we use to discover available paths for transmiting data information, are used as well for service discovery.

\section{SERVICE DISCOVERY MECHANISM}

The design of the OLSR protocol ${ }^{5}$ helps us to add extensions to the protocol through the addition of new messages types. We have defined a new message type into OLSR called Service Discovery message (SDM). Figure 1 illustrates the message format:

\begin{tabular}{|c|c|c|c|}
\hline \multirow{2}{*}{$\begin{array}{r}\text { Birs: } \\
\text { MESSAGE }\end{array}$} & \multicolumn{3}{|c|}{ 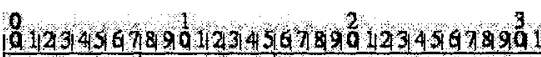 } \\
\hline & The T & Lve Hog Count & Mersage sequence Number \\
\hline & Type & service Name & SOM INTERVAL \\
\hline & \multicolumn{3}{|c|}{ Liferime service Describtian } \\
\hline
\end{tabular}

Figure 1. SDM packet format

The Time To Live field determines the number of hops the message can travel. This let us save more bandwidth than with a standard broadcast-based solution. The Hop Count field contains the number of hops a message is attaining. The Type indicates whether the message is an adverstisement or a query request. The LifeTime field indicates the time the service advertisement will be available for the rest of the nodes. Finally, the SDM INTERVAL is a parameter that indicates how often an advertisement message is transmited. It is an user configurable parameter, so depending on the working environment this parameter could be higher or smaller. The smaller it is the more overhead it will be introduced into the network.

\subsection{Service cache}

Service cache enables devices to store their local and foreign services. Each node, when receiving an advertisement, stores the service information in the service cache in the room fitted-out for that purpose. 
The cache size will increase with the number of services. Once the cache is full, the first entries to be discarded will be the ones with the lowest remaining LifeTime. Also the entries with the lifetime expired will be automatically removed.

\subsection{Service advertisements}

Every SDM INTERVAL each node will send a new message with the update services. In case there are no new services available the node will not piggyback the message into the OLSR header. The way a node verifies whether it hosts new services or not is by checking the number of entries it has in its local cache where it stores its local services.

Any mobile device hearing this advertisement and interested in the services will store them in its service cache.

It is possible that two different nodes advertise the same service. As the service cache of each device is finite, before storing the services, a node that receives it will first check whether the service offering has been already advertised by another node. If so, it will discard the advertisement.

\subsection{Service discovery}

The format of the service discovery message is the same as that of the service advertisement, which is illustrated in Figure 1.

MPR nodes will forward the request. A node receiving the request will check whether the service wanted match one of the ones that it supports and it will make a replay. Before, it will wait for a random time to verify whether there is already a reply message from other node. If affirmative, it ignores the service query message. This way the protocol avoids multiple reply copies of the same service being sent at the same time to save network bandwidth. Otherwise, it fills in the fields of the SD message such as Time to Live, Server Address, Service Port, Protocol Type, and the Option field.

\section{SIMULATION RESULTS}

\subsection{Simulation environment}

The ns $-2^{7}$ simulation tool is used to evaluate the protocol. In the simulations, each mobile node changes its location within the subnet based on the random-waypoint ${ }^{8}$ model. In $\mathrm{ns}-2$, the Distributed Coordination 
Function (DCF) of IEEE 802.11 for wireless LANs is used as the MAC layer protocol.

The simulated network is comprised of 50 nodes. Each scenario runs for 900 seconds. We use 3 different SDM frequency values: $0,5 \mathrm{~s}, 1,5 \mathrm{~s}$ and $2,5 \mathrm{~s}$.

\subsection{Simulation results}

We study for various SDM frequency intervals the traffic overhead in packets per second. In the following figure we show the overhead introduced by the SDM messages embedded in the OLSR protocol:

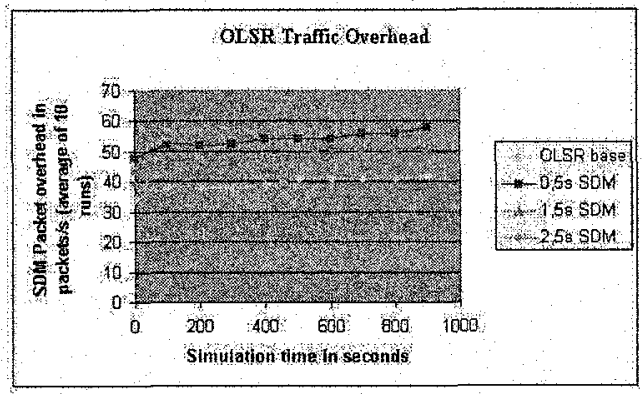

Figure2. SDM packet overhead in the network (in packets/s)

We can observe that the traffic overhead generated by the SDM messages when we advertise them for different frequencies is practically the same as in the basic OLSR protocol. Thus, we can conclude that the traffic overhead introduced with our service discovery proposal is not significant and it can be applied as a generic service discovery mechanism with the OLSR protocol and a mobile environment.

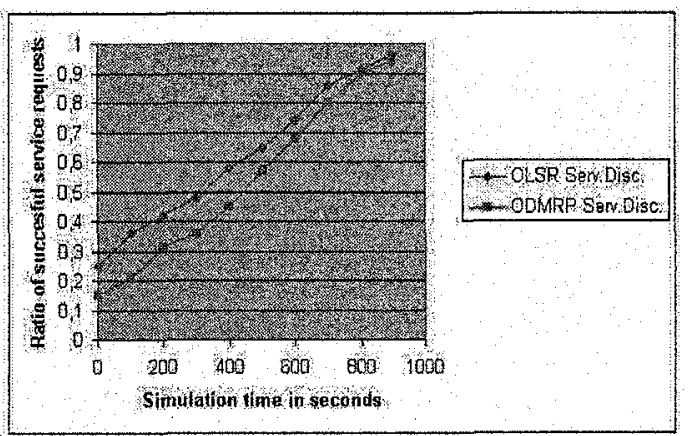

Figure3. Ratio of successful request 
Regarding the ratio of service requests, in the figure above are depicted the results we have obtained.

We have compared the results with the service discovery mechanism over the reactive protocol ODMRP. From the figure we conclude that the service discovery made over the proactive protocol is more effective than the one over a reactive protocol, in terms of response time in service requests. The reason is that in a proactive protocol we have more knowledge or "world-view" of the network, and the routes for transmitting the packages are known in advance.

\section{CONCLUSION AND FUTURE WORK}

In this paper, we propose a service discovery mechanism for proactive ad hoc networks, based on the OLSR routing protocol. The service discovery information is piggybacked in the OLSR protocol as a new message type called SDM.

The simulation results have shown that the proposed mechanism is valid for ad hoc mobile networks. We are currently checking how the protocol behaves when the service cache is full, how it deals with the unavailability of services and false service discoveries. In a second phase of our project we will consider security aspects in the protocol.

\section{REFERENCES}

1. E. Guttman, C. Perkins, J. Veizades, and M. Day, "Service Location Protocol, Version 2", RFC 2608, June 1999.

2. Edwards and T. Rodden. Jini Example by Example. Prentice Hall PTR, June 2001.

3. John. UpnP, Jini and Salutation- A Look at some popular Coordination Frameworks for Future Network Devices. Technical report, California Software Labs, 1999.

4, Bluetooth Specification Part E. Service Discovery Protocol (SDP). http://www bluetooth.com, November 1999.

5. T. Clausen and P.Jacquet "Optimized Link State Routing Protocol (OLSR". RFC 3626, IETF Network Working Group, October 2003.

6. http://www.ietf.org/html.charters/manet-charter.html, work in progress.

7. The Network Simulator ns-2 Homepage. http://www.isi.edu/nsnam/ns.

8. David B. Johnson and David A. Maltz, Dynamic Source Routing in ad hoc Wireless Networks, in Mobile Computing, edited by Tomas imielinski and Hank Korth, Chapter 5, pages 153-181, Kluwer Academic Publishers, ISBN:0792396979, 1996. 